\title{
Towards Optimal Central Venous Catheter Tip Position
}

\section{Schummer $\mathbf{W}^{1,2 *}$}

${ }^{1}$ Clinic for Anesthesiology and Intensive Care Medicine, Friedrich Schiller University, Jena, Germany

${ }^{2}$ Ruhrlandklinik West German Lung Center, University Hospital Essen, Essen, Germany

\begin{abstract}
Central venous catheters (CVCs) are required in many critically ill patients. As with most invasive procedures central venous catheterisation is associated with numerous potential complications, many of which stem from the access procedure. Other serious complications are related to the catheter tip position. Secondary to CVC misplacement, there is significant morbidity and mortality associated with complications such as perforation of the vessel, thrombosis, extravasation and arrhythmias with its related consequences. In clinical practice, methods used to determine CVC tip position are chest radiography and ECG guidance of the CVC during the insertion procedure.
\end{abstract}

Keywords: Central venous catheterization; Complications; Perforation; Thrombosis; Extravasation; Arrhythmias; ECG-guidance; Chest radiography; Interventional radiology; Vascular surgery

\section{Introduction}

Many critically ill patients require central venous catheters (CVCs) [1]. Usually CVCs are inserted via the subclavian or internal jugular vein. As with most invasive procedures, CVC usage has numerous potential complications, many of which stem from the insertion procedure [2-4]. Ultrasound guidance during CVC insertion has been shown to significantly improve patient safety $[5,6]$.

In addition to insertion complications, the optimal CVC tip position remains the subject of debate because no position is considered to be absolutely safe [7]. Despite the level of skill of the operator and the use of ultrasound guidance, central venous catheter (CVC) placement can result in CVC malpositioning, an unintended placement of the catheter tip in an inadequate vessel or position $[4,8]$.

\section{Perforation}

Venous or right heart perforations, resulting in pericardial tamponade or hemothoraces, have a $65-95 \%$ mortality rate [1]. These perforations can be caused by a misplaced CVC tip. Unfortunately, current literature does not contain sufficient studies that address the relationship between specific tip placement and specific complications.

Due to pericardial tamponade risks, the US Food and Drug Administration (FDA) strongly advises that the CVC tip should not be placed in, or be allowed to migrate into, the heart [9]. Apart from improper catheter placement, especially when using hemodialysis catheters) [10], at a too proximal tip position, patients are prone to extravasation injury and vessel thrombosis [11]. These and resultant complications are a real threat to patients. The incidence of vessel perforation appears to be approximately 0.25 to $0.4 \%$, but there are no reliable data on post-placement incidences of cardiac tamponade, or any other CVC-position related complication [1].

\section{Thrombosis}

Thrombosis is not a benign, self-limited condition. It can cause serious complications, e.g. pulmonary embolism, loss of vascular access, superior vena cava syndrome. Contributing factors include venous access route, catheter material biocompatibility, catheter length and size, and time in place. Other factors promoting venous thrombosis include endothelial damage through the CVC, hypercoagulable states, inflammation and infection.
When a catheter causes injury to the vein wall, a clot forms at the lesion point and a sleeve can form as early as $24 \mathrm{~h}$ after catheterization. The clot then transforms into vascularized connective tissue after 5-14 days. The sleeve is mainly formed by smooth muscle cells migrating from the injured vein wall into the early pericatheter thrombus [12]. The sleeve is permanently attached to the vein wall, making it a very unlikely cause of embolism. Other reports have shown that these fibrin sleeves may, in rare cases, cause pulmonary embolism spontaneously or after fibrin sheath stripping $[12,13]$. Thrombosis, as well as fibrin sheath formation play a central role in causing CVC dysfunction.

Studies on silicone catheters show a relationship between thrombosis and tips placed higher than, or high within, the superior vena cava. The morbidity and mortality rates caused by such thrombus may exceed those caused by perforation. Studies on polyurethane catheters show a high risk of both thrombosis and sleeve formation.

\section{Extravasation}

Extravasation is an unintentional injection or leakage of fluid in the perivascular or subcutaneous space [14]. Extravasation injury results from a combination of factors, including solution cytotoxicity, osmolality, vasoconstrictor properties, infusion pressure, regional anatomical peculiarities, and other patient factors and may be associated with tissue injury [14]. Extravasation is less frequent in CVCs than in peripheral lines, but potentially more dangerous because it is more difficult to detect and is more likely to involve vulnerable anatomical structures [15]. When using a multilumen catheter, it is particularly important to be aware of the position of the ports relative to the tip and the total length of the catheter. Depending on insertion depth, the extravasal position of the proximal port can occur when a multilumen catheter is inadvertently withdrawn just a few centimeters [15].

${ }^{*}$ Corresponding author: Schummer W, Ruhrlandklinik West German Lung Center, University Hospital Essen, Tuschener Weg 40, 45239 Essen, Germany, Tel: +49 201 7230; E-mail: cwsm.schummer@gmx.de

Received February 25, 2016; Accepted March 17, 2016; Published March 26 2016

Citation: Schummer W (2016) Towards Optimal Central Venous Catheter Tip Position. J Vasc Med Surg 4: 260. doi:10.4172/2329-6925.1000260

Copyright: $\odot 2016$ Schummer W. This is an open-access article distributed unde the terms of the Creative Commons Attribution License, which permits unrestricted use, distribution, and reproduction in any medium, provided the original author and source are credited. 


\section{Arrhythmias}

Contact of a guide wire or catheter with the endocardium may induce arrhythmias or heart block. When CVCs are placed without imaging guidance, a significant number of patients experience atrial or ventricular dysrhythmias $41 \%$ and $25 \%$, respectively. Such dysrhythmias are usually transient and asymptomatic; however, in patients suffering from severe aortic stenosis, acute myocardial ischemia, or left bundle branch block, dysrhythmias may be life-threatening.

\section{Methods To Determine CVC Tip Position}

Verifying catheter tip position is not particularly easy. In the following section traditional and recent methods and their pitfalls are delineated.

Since the first placement by Forßmann in 1929, chest radiography has been used to verify CVC position. Today, the American College of Radiology recommends portable radiographs for critically ill patients following placement of support and monitoring devices such as endotracheal tubes, CVCs, etc $[4,8]$. Bedside chest radiography differs from standard erect radiography in several critical respects. It has by far greater variability in positioning and technique (e.g. due to rotation, lordotic or reverse lordotic positioning, extent of lung inspiration). This has profound effects on the interpretation of the radiograph. Due to inherent projection phenomena, however, it should not be used to assess tip position with respect to the right atrium (RA) [16].

ECG-guidance is a reliable method to use for patients with sinus rhythm. Many clinical studies have shown that ECG-guidance during CVC placement is also an adequate tool to prevent malpositions $[17,18]$. A safe method for ECG-guidance of the CVC follows (using CVC kits from B.Braun, Melsungen, Germany): Insert the guide wire in the vein to a depth not to exceed $10-12 \mathrm{~cm}$. Introduce the catheter over the wire, up to the black marking that indicates the point at which the tip of the wire levels with the distal port opening (Figure 1) [19]. To make sure that this method works a sterile connection cable with a crocodile clip has to be clamped to the guide wire [19]. The other end of the connection cable joins a switch box (e.g. Certodyn universal adaptor, B. Braun Melsungen AG, Germany) which has to be joined up in the circuit. This enables to switch the right arm ECG electrode to the catheter tip to get an intravascular ECG displayed. Then carefully and simultaneously advance the catheter and the guide-wire under ECGguidance until an increase in $\mathrm{P}$ wave size is detected. The first increase in $\mathrm{P}$ wave amplitude (Einthoven lead II) relates to the pericardial reflection (Figure 2). This is the only bedside method which provides reliable identification of the pericardial reflection, and thereby, consistently correct placement of the CVC outside the pericardium [11]. The $P$ wave amplitude increases to its maximum once the catheter tip levels with the superior vena cava/right atrial junction (SVC/RA) [11]. Further advancement of the catheter tip results in a decrease in $P$ wave amplitude or biphasic $\mathrm{P}$ wave. This has been confirmed by several studies using transesophagel echocardiography (TEE).

ECG guidance is not capable of providing information regarding the angle of CVCs to the vessel wall or whether the CVC inadvertently had been placed arterial. We suggest placing the CVC tip at the maximal $\mathrm{P}$ wave amplitude, as CVCs typically end in the lower SVC at junction with the right atrium. At this position catheters - even those inserted from the left - will run parallel to the vessel wall $[8,18]$.

Although plain chest radiography is the standard imaging modality for confirming catheter tip location, signs and symptoms of CVC malpositioning even in presence of normal or inconclusive conventional radiography findings should prompt the use of additional diagnostic methods to confirm or rule out CVC malpositioning $[4,8]$. With very few exceptions, the recommendation in cases of intravascular CVC malpositioning is to remove and relocate the catheter $[4,8]$.

\section{Unintended Arterial Cannulation}

Early recognition of the arterial trauma and prompt management are required [20]. Two different approaches to unintended arterial cannulation during central venous catheterization have been taken: removal of the cannula, followed by the application of local pressure, and immediate surgical or endovascular management [20,21].

The present data demonstrate that with catheter $7 \mathrm{~F}$ and over, the pull/pressure technique could be associated with significantly higher morbidity than surgical or endovascular management, including stroke, suddenly expanding hematoma causing airway compression, false aneurysm, or death [20-22]. Under no circumstances should prolonged arterial cannulation be tolerated [20]. Several cases are described with thrombus found at the site of the arterial injury, especially after prolonged catheterization. Heparinization should be considered if immediate treatment is not possible [20,23].

After arterial repair, prompt neurological evaluation should be performed, even if it requires postponing elective intervention [20-22]. Imaging is suggested to exclude arterial complications, especially if arterial trauma site was not examined and repaired [20].

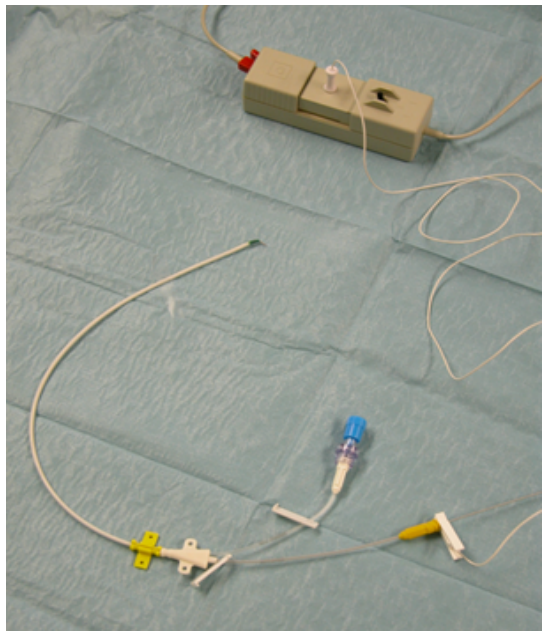

Figure 1: ECG-guidance during CVC insertion. Attach connection cable (white) with a crocodile clip to the guide wire. The other end of the connection cable joins a switch box which has to be joined up in the circuit. This enables to switch the right arm ECG electrode to the catheter tip to get an intravascular ECG displayed. Arrow indicates tip of the guide wire which serves as an intravascular electrode.

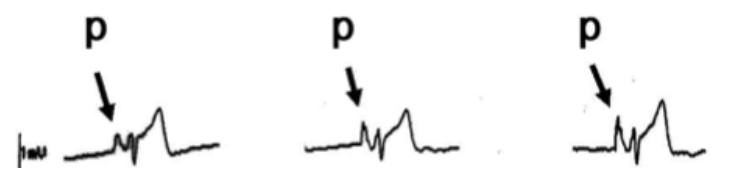

Figure 2: ECG-guidance during insertion: a) Normal $P$ wave amplitude in $\mathrm{V}$. superior vena cava (SVC) above pericardial reflection. b) At pericardial reflection first significant increase in $P$ wave amplitude. c) Maximal $P$ wave amplitude at SVC/right atrium junction. 


\section{Conclusion}

Placement of a CVC is always associated with risks [11]. Ultrasound guidance during $\mathrm{CVC}$ insertion has been shown to significantly improve patient safety.

Clinicians should attempt to reduce risk by placing CVCs so that they function properly and where the risk of perforation is low, i.e. no angles $>40^{\circ}$ to the vessel wall [11]. The optimal position is close to the superior vena cava/ right atrial junction and can be specifically determined by using ECG-guidance to identify the maximal $\mathrm{P}$ wave amplitude $[11,24]$. In clinical practice, radiologists and intensivists typically use bedside CXR, the least sophisticated imaging procedure available, to decide CVC tip placement. Although bedside CXR does not compare to TEE for assessing CVC tip position, particularly with regard to the RA, CXR remains a valuable tool to identify other CVC malpositions or complications, such as pneumothorx - and it is widely available.

\section{Key Messages}

- Central venous catheterisation is associated with numerous potential complications, many of which stem from the access procedure $[1,20]$. Other serious complications are related to the catheter tip position.

- Clinicians should attempt to reduce risks by placing CVCs under ultrasound guidance.

- CVC's should function properly and should be placed at a position where the risk of perforation is low.

- The optimal position is close to the superior vena cava/right atrial junction and can be specifically determined by using ECG guidance during the access procedure.

- Methods used in clinical practice to determine CVC tip position are chest radiography and ECG guidance.

- Although not ideal for CVC tip assessment (post-hoc monitoring, projection phenomena), chest radiography remains a valuable tool to identify other CVC malpositions or complications $[4,25]$.

- Unintended arterial cannulation should be detected and managed as fast as possible. If arterial trauma with a large-caliber catheter occurs, prompt surgical or endovascular treatment seems to be the safest approach [20]. After arterial repair, prompt neurological evaluation should be performed, even if it requires postponing elective intervention [20-22].

\section{References}

1. Schummer W, Sakr Y, Schummer C (2008) Towards Optimal Central Venous Catheter Tip Position. Yearbook of Intensive Care and Emergency Medicine Vincent J-L: 581-590.

2. Fazeny-Dörner B, Wenzel C, Berzlanovich A, Sunder-Plassmann G, Greinix $\mathrm{H}$, et al. (2003) Central venous catheter pinch-off and fracture: recognition, prevention and management. Bone Marrow Transplant 31: 927-930.

3. Chirinos JC, Neyra JA, Patel J, Rodan AR (2014) Hemodialysis catheter insertion: is increased $\mathrm{PO} 2$ a sign of arterial cannulation? A case report. BMC Nephrol 15: 127

4. Roldan CJ, Paniagua L (2015) Central Venous Catheter Intravascular Malpositioning: Causes, Prevention, Diagnosis, and Correction. West J Emerg Med 16: 658-664.

5. Schummer W, Köditz JA, Schelenz C, Reinhart K, Sakka SG (2014) Preprocedure ultrasound increases the success and safety of central venous catheterizationâ€. Br J Anaesth 113: 122-129.
6. Lamperti M, Bodenham AR, Pittiruti M, Blaivas M, Augoustides JG, et al. (2012) International evidence-based recommendations on ultrasound-guided vascular access. Intensive Care Med 38: 1105-1117.

7. Fletcher SJ, Bodenham AR (2000) Safe placement of central venous catheters: where should the tip of the catheter lie? Br J Anaesth 85: 188-191.

8. Nama RK, Bhosale GP, Shah VR (2015) Idiopathic unilateral hypoplasia of internal jugular vein and coagulopathy: Unusual case for central venous catheterization. Anesth Essays Res 9: 437-439.

9. Bayer O, Schummer C, Richter K, Fröber R, Schummer W (2006) Implication of the anatomy of the pericardial reflection on positioning of central venous catheters. J Cardiothorac Vasc Anesth 20: 777-780.

10. Vesely TM (2003) Central venous catheter tip position: a continuing controversy J Vasc Interv Radiol 14: 527-534.

11. Bayer O, Schummer C, Richter K, Fröber R, Schummer W (2006) Implication of the anatomy of the pericardial reflection on positioning of central venous catheters. J Cardiothorac Vasc Anesth 20: 777-780.

12. Oguzkurt L, Tercan F, Torun D, Yildirim T, Zümrütdal A, et al. (2004) Impact of short-term hemodialysis catheters on the central veins: a catheter venographic study. Eur J Radiol 52: 293-299.

13. Winn MP, McDermott VG, Schwab SJ, Conlon PJ (1997) Dialysis catheter 'fibrin-sheath stripping': a cautionary tale! Nephrol Dial Transplant 12: 10481050.

14. Schummer W, Schummer C, Bayer O, Müller A, Bredle D, et al. (2005) Extravasation injury in the perioperative setting. Anesth Analg 100: 722-727, table of contents.

15. Schummer C, Sakr Y, Steenbeck J, Gugel M, Reinhart K, et al. (2010) Risk of extravasation after power injection of contrast media via the proximal port of multilumen central venous catheters: case report and review of the literature. Rofo 182: 14-19.

16. Wirsing M, Schummer C, Neumann R, Steenbeck J, Schmidt P, et al. (2008) Is traditional reading of the bedside chest radiograph appropriate to detect intraatrial central venous catheter position? Chest 134: 527-533.

17. McGee WT, Ackerman BL, Rouben LR, Prasad VM, Bandi V, et al. (1993) Accurate placement of central venous catheters: a prospective, randomized multicenter trial. Crit Care Med 21: 1118-1123.

18. Kremser J, Kleemann F, Reinhart K, Schummer W (2011) Optimized method for correct left-sided central venous catheter placement under electrocardiographic guidance. Br J Anaesth 107: 567-572.

19. Karaaslan D, Altinisik U, Peker TT, Nayir E, Ozmen S (2009) External jugular vein catheterization using 'intra-atrial electrocardiogram'. Yonsei Med J 50 : 222-226.

20. Guilbert MC, Elkouri S, Bracco D, Corriveau MM, Beaudoin N, et al. (2008) Arterial trauma during central venous catheter insertion: Case series, review and proposed algorithm. J Vasc Surg 48: 918-925.

21. Yamamoto A, Suzuki K, Sakaida H, Suzuki H, Imai H (2015) Management of inadvertent vertebral artery injury due to central venous catheterization in a coagulopathic patient. Acute Medicine \& Surgery.

22. Kwon OS, Lee HJ, Kim WS, Hong JM, Cho HJ (2014) Risk of continuing planned surgery after endovascular repair of subclavian artery injury: a case report. Korean J Anesthesiol 67: 139-143.

23. Reuber M, Dunkley LA, Turton EP, Bell MD, Bamford JM (2002) Stroke after internal jugular venous cannulation. Acta Neurol Scand 105: 235-239.

24. Chirinos JC, Neyra JA, Patel J, Rodan AR (2014) Hemodialysis cathete insertion: is increased PO2 a sign of arterial cannulation? A case report. BMC Nephrol 15: 127.

25. Schummer W, Schummer C, Rose N, Niesen WD, Sakka SG (2007) Mechanica complications and malpositions of central venous cannulations by experienced operators. A prospective study of 1794 catheterizations in critically ill patients. Intensive Care Med 33: 1055-1059. 